University of Wollongong

Research Online

Australian Institute for Innovative Materials -

Papers

Australian Institute for Innovative Materials

$1-1-2015$

Inkjet-printed alginate microspheres as additional drug carriers for injectable hydrogels

Johnson Chung

University of Wollongong, johnsonc@uow.edu.au

Sina Naficy

University of Wollongong, snaficy@uow.edu.au

Gordon G. Wallace

University of Wollongong, gwallace@uow.edu.au

Stephen O'Leary

University of Melbourne

Follow this and additional works at: https://ro.uow.edu.au/aiimpapers

Part of the Engineering Commons, and the Physical Sciences and Mathematics Commons

Research Online is the open access institutional repository for the University of Wollongong. For further information contact the UOW Library: research-pubs@uow.edu.au 


\title{
Inkjet-printed alginate microspheres as additional drug carriers for injectable hydrogels
}

\begin{abstract}
Local delivery of bioactive molecules to the inner ear via diffusion through the round window membrane is becoming an attractive approach to treat sensorineural hearing loss compared to systemic drug administration. Pluronics ${ }^{\circledR}$ (Lutrol F127) are a class of thermosensitive hydrogels that remain liquid prior to injection and rapidly gel under physiological conditions. They are, however, limited to short-term drug release due to rapid hydrolysis in aqueous solution. Therefore, the aim of this study was to investigate an approach, using an ink-jet printing system, to sustain the drug release by incorporating hydrogel microspheres within Lutrol F127. Various concentrations of Lutrol F127 and calcium chloride ( $\mathrm{CaCl} 2)$ were examined by rheology to determine the optimum combinations to use for injection and then blended with inkjet-printed alginate microspheres. Drug release (FITC-Dextran) from Lutrol F127 alone reached completion in less than $24 \mathrm{~h}$. Release from alginate spheres alone showed a burst release and reached $100 \%$ in $6 \mathrm{~h}$. Interestingly, the incorporation of microspheres within Lutrol F127 allowed a more sustained release profile and a slower burst release. By varying the quantity of microspheres, concentration of alginate, or ionic cross-linking ratio, the release profile can be adjusted to suit the desired application.
\end{abstract}

\section{Keywords}

drug, carriers, injectable, hydrogels, printed, inkjet, alginate, microspheres, additional

Disciplines

Engineering | Physical Sciences and Mathematics

\section{Publication Details}

Chung, J. H. Y., Naficy, S., Wallace, G. G. \& O'Leary, S. (2016). Inkjet-printed alginate microspheres as additional drug carriers for injectable hydrogels. Advances in Polymer Technology, 35 (4), 439-446. 


\title{
Inkjet-Printed Alginate Microspheres as Additional Drug Carriers for Injectable Hydrogels
}

\author{
JOHNSON H. Y. CHUNG, SINA NAFICY, GORDON G. WALLACE \\ Intelligent Polymer Research Institute, Australian Research Council Centre of Excellence for Electromaterials Science, University of Wollongong, \\ Wollongong, NSW 2522, Australia
}

\author{
SINA NAFICY \\ School of Mechanical, Materials and Mechatronic Engineering, University of Wollongong, Wollongong, NSW 2522, Australia
}

\section{STEPHEN O'LEARY}

Department of Otolaryngology, The University of Melbourne, Royal Victorian Eye and Ear Hospital, East Melbourne, VIC 3002, Australia

Correspondence to: Gordon G. Wallace; e-mail: gwallace@uow.edu.au

Received: January 21, 2015

Accepted: July 23, 2015

\begin{abstract}
Local delivery of bioactive molecules to the inner ear via diffusion through the round window membrane is becoming an attractive approach to treat sensorineural hearing loss compared to systemic drug administration. Pluronics ${ }^{\circledR}$ (Lutrol F127) are a class of thermosensitive hydrogels that remain liquid prior to injection and rapidly gel under physiological conditions. They are, however, limited to short-term drug release due to rapid hydrolysis in aqueous solution. Therefore, the aim of this study was to investigate an approach, using an ink-jet printing system, to sustain the drug release by incorporating hydrogel microspheres within Lutrol F127. Various concentrations of Lutrol F127 and calcium chloride $\left(\mathrm{CaCl}_{2}\right)$ were examined by rheology to determine the optimum combinations to use for injection and then blended with inkjet-printed alginate microspheres. Drug release (FITC-Dextran) from Lutrol F127 alone reached completion in less than $24 \mathrm{~h}$. Release from alginate spheres alone showed a burst release and reached $100 \%$ in $6 \mathrm{~h}$. Interestingly, the incorporation of microspheres within Lutrol F127 allowed a more sustained release profile and a slower burst release. By varying the quantity of microspheres, concentration of alginate, or ionic cross-linking ratio, the release profile can be adjusted to suit the desired application. (c) 2015 Wiley Periodicals, Inc. Adv Polym Technol 2016, 35, 21571; View this article online at wileyonlinelibrary.com. DOI 10.1002/adv.21571
\end{abstract}

KEY WORDS: Alginate, Biopolymers, Hydrogels, Drug delivery systems, Lutrol F127

\section{Introduction}

0 ensorineural hearing loss results from damage at any point between the cochlea hair cells and the auditory cortex in the brain, most commonly at the hair cells. ${ }^{1}$ As neurons and hair cells in humans do not regenerate spontaneously, majority of treatment options were more focused toward functional improvements (i.e., hearing aids and cochlear implants) rather than a medical treatment. ${ }^{2,3}$ However, local delivery of bioactive molecules intratympanically (diffusion across the round window membrane) is becoming a major approach to protecting the neurosensory structures of the inner ear after injury, as conventional systemic drug administration may be less effective due to physiological and anatomical barriers such as the bloodcochlea barrier that limit drug levels within the inner ear. ${ }^{4-7}$ Local therapy to the inner ear is also ideally suited to the administration of therapeutics that may be toxic when administered systemically, and in this regard trophic agents or antiapoptotic agents are under investigation.

Using injectable hydrogels to deliver bioactive molecules have become an attractive method compared to traditional implantable drug delivery systems as these minimize surgical intervention and mostly can be prepared simply via physical mixing. Injectable hydrogels can be formed in situ by either chemical polymerization or sol-gel phase transition. The sol-gel transition systems remain liquid prior to injection, but rapidly gel under physiological conditions. ${ }^{8}$ This means that these materials can adapt to any shape within the target site and provide a uniform distribution of bioactive molecules at the injection site once gelled. Pluronics ${ }^{\circledR}$ (Lutrol F127) are a class of commercially available thermosensitive hydrogels that have been widely reported as injectable hydrogels in tissue engineering applications. ${ }^{9}$ At moderate concentrations (15$30 \% \mathrm{w} / \mathrm{w})$, these triblock copolymers of poly(ethylene oxide)poly(propylene oxide)-poly(ethylene oxide) (PEO-PPO-PEO) 


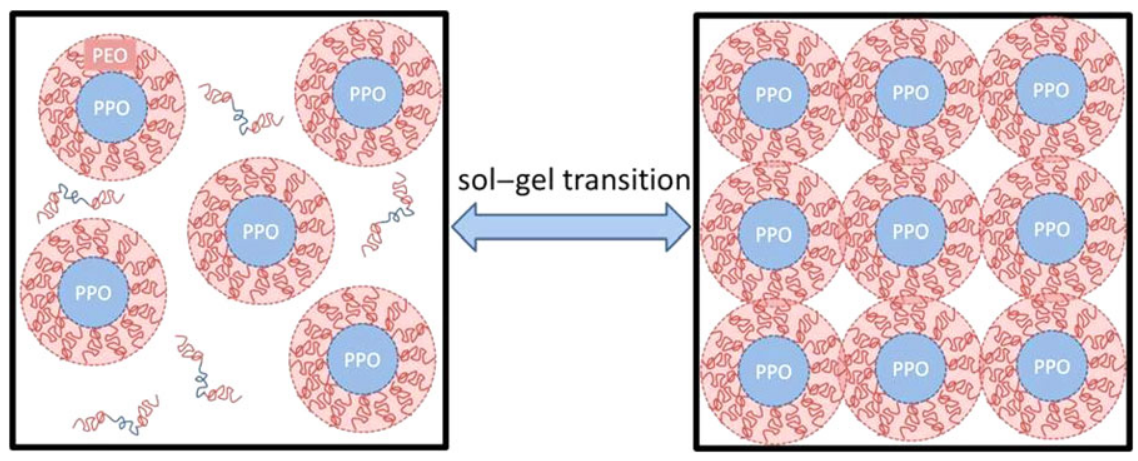

FIGURE 1. Schematic diagram of sol-gel transition of Lutrol F127 aqueous solution upon reaching a critical concentration and temperature.

undergo a reversible thermal transition in aqueous solution under physiological temperature and $\mathrm{pH}$, as shown in Fig. $1 .{ }^{10,11}$ However, the major drawback of the system is the rapid erosion of the gel by body fluids, thereby limiting its long-term drug release. ${ }^{12}$ Attempts to prolong drug release can include additives or blending of polymers, ${ }^{13,14}$ changing size or hydrophobicity of the drug to be released, ${ }^{15}$ or via the addition of a second drug carrier system. ${ }^{11,16}$ Here, we will adapt on the later approach by using a nozzle-based ink-jetting system to fabricate alginate microspheres that will act as a secondary drug carrier system once embedded within Lutrol F127.

Nozzle-based ink-jetting system has been long favored in the fabrication of microspheres due to its good size distribution, controllability, and scale-up potential. ${ }^{17,18}$ On the other hand, alginate is known for its cell compatibility and good stability. ${ }^{19}$ Therefore, the objective of this study was to evaluate the feasibility of incorporating alginate hydrogel microspheres into the thermosensitive gel (Lutrol F127) for sustained intratympanic delivery of FITC-dextran as the model drug. In addition, the use of inkjet printing as a simple and alternative method to fabricate hydrogel microspheres will be assessed.

\section{Materials and Methods}

\section{MATERIALS}

Poloxamer 407 (Lutrol F127), alginic acid sodium salt (viscosity of $100-300 \mathrm{cP}$ for $2 \mathrm{wt} \%$ solution, $25^{\circ} \mathrm{C}$ ), and model drug (FITC-dextran $20 \mathrm{kDa}$ ) were obtained from Sigma-Aldrich, Australia. Other reagents were all analytical grade and used as received.

\section{LUTROL F127 INK FORMULATION}

Lutrol F127 solutions ( $\% \mathrm{w} / \mathrm{v})$ were prepared in saline $(0.9 \%$ $\mathrm{NaCl})$ using the cold method. ${ }^{20}$ In brief, appropriate quantities of Lutrol F127 were slowly added to a cold buffer solution and stirred until a clear, homogeneous liquid formed. The gel formulations were kept under refrigeration $\left(4^{\circ} \mathrm{C}\right)$ and thoroughly stirred before experiments.

\section{FABRICATION OF ALGINATE MICROSPHERES USING INKJET PRINTING SYSTEM}

Printing was conducted using a piezoelectric Dimatix Materials Printer 2800 (DMP 2800m; Fujifilm, Santa Clara, CA), equipped with a 16 nozzle $10 \mathrm{pL}$ cartridge (DMCLCP-11610) at a drop spacing of $50 \mu \mathrm{m}$ and height from $18 \mathrm{~mm}$. Alginate solution at $0.5 \% \mathrm{w} / \mathrm{v}$ was printed into a cross-linking bath of 1,2 , or $5 \% \mathrm{w} / \mathrm{v} \mathrm{CaCl} \mathrm{Cl}_{2}$ to determine the optimum concentration of crosslinking concentration to obtain consistent hydrogel spheres. The printed alginate microspheres were imaged using a Leica DMIL LED microscope (Leica Microsystems, Wetzlar, Germany) and the bead diameter analyzed using Image processing software, Image J.

\section{PREPARATION OF MICROSPHERE-LOADED INJECTABLE HYDROGELS}

A schematic diagram of the preparation process is shown in Fig. 2. In brief, alginate $(0.5 \% \mathrm{w} / \mathrm{v})$ in saline $(0.9 \% \mathrm{NaCl})$ was loaded into the cartridge and printed into a cross-linking solution of $2 \% \mathrm{w} / \mathrm{v} \mathrm{CaCl} 2$ and $10 \mu \mathrm{g} / \mathrm{mL}$ FITC-dextran. A total of 100,000 or 500,000 microspheres $/ \mathrm{mL}$ were printed into the cross-linking solution. The solution was kept away from light for 1 day for the alginate microspheres to reach equilibrium. From the solution, appropriate amounts of Lutrol F127 were added to obtain a final concentration of $20 \% \mathrm{w} / \mathrm{v}$ Lutrol F127,

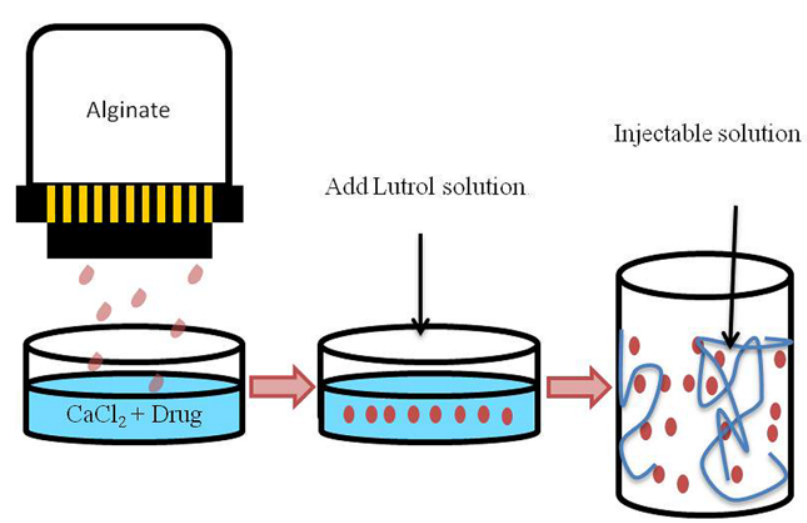

FIGURE 2. Schematic diagram of preparing the microsphere-loaded injectable delivery system. 
$0.5 \% \mathrm{w} / \mathrm{v} \mathrm{CaCl}_{2}$, and $2 \mu \mathrm{g} / \mathrm{mL}$ drug loading. This concentration was used based on a series of experiments and discussed in Sections Rheology and Microsphere Fabrication. A final drug loading of $2 \mu \mathrm{g} / \mathrm{mL}$ was selected based on the standard curve conducted (not shown) showing that this loading would fit well within the detection limit of the instrument.

\section{RHEOLOGY}

The rheological behavior of Lutrol F127 formulations was analyzed using an AR-G2 rheometer (TA Instruments, New Castle, DE) equipped with a Peltier plate thermal controller. A $2^{\circ} / 40 \mathrm{~mm}$ cone and plate geometry was used in all measurements. The solutions were allowed to reach the equilibrium temperature for 1 min prior to performing the experiments.

Storage modulus $\left(G^{\prime}\right)$ and loss modulus $\left(G^{\prime \prime}\right)$ were measured as a function of temperature (at a constant frequency). Temperature sweep experiments were conducted at a rate of $2^{\circ} \mathrm{C} / \mathrm{min}$ from 7 to $70^{\circ} \mathrm{C}$ at a fixed strain and frequency of $1 \%$ and $1 \mathrm{~Hz}$, respectively. Viscosity was measured as a function of temperature at a constant shear rate. Temperature ramp experiments were conducted at a ramp rate of $1^{\circ} \mathrm{C} / \mathrm{min}$ from 5 to $70^{\circ} \mathrm{C}$ at a shear rate of $1 / \mathrm{s}$.

\section{IN VITRO DRUG RELEASE STUDY}

Drug release studies for Lutrol F127 and its formulations with microspheres were conducted using a membrane-less method in saline at $37^{\circ} \mathrm{C}$. Materials $(1 \mathrm{~mL})$ were cast directly into preweighed glass vials of $1 \mathrm{~cm}$ inner diameter. Glass vials were kept in a $37^{\circ} \mathrm{C}$ incubator until the material has gelled. The initial weight of samples was recorded and a sink volume of $1 \mathrm{~mL}$ saline was added to the vials and placed in an incubator on an orbital shaker at $60 \mathrm{rpm}$. At regular time intervals, the release medium was temporarily removed and small aliquots $(200 \mu \mathrm{L})$ were taken to be measured by a fluorescent reader (FLUOstar Omega, Offenburg, Germany). The weight of the glass vial and gel was recorded to calculate the mass loss of the gel and the release medium was then added back into the vials. The readings were taking at an excitation wavelength of $490 \mathrm{~nm}$ and emission of $520 \mathrm{~nm}$. The assay was repeated in triplicate and each assay consisted of a triplicate of each material.

For drug release from microspheres, appropriate amounts of alginate and the model drug were prepared in saline and loaded into the printer cartridge. These were calculated to yield the same total drug loading as Lutrol F127 and Lutrol F127 microspheres. $\mathrm{CaCl}_{2}$ cross-linking solution was placed beneath the inkjet cartridge. Printed microspheres were immediately transferred into glass vials and drug release was conducted similarly as mentioned above.

\section{DRUG RELEASE KINETICS AND MECHANISM STUDY}

Release kinetics were evaluated according to the following models: zero-order kinetics (Eq. (1)), first-order kinetics (Eq. (2)), and Higuchi's square root of time (Eq. (3)). ${ }^{11,21}$

$$
M=k_{0} t
$$

$$
\begin{gathered}
\ln M_{t}=\ln M_{0}-k_{1} t \\
M=k_{H} \sqrt{t}
\end{gathered}
$$

where $M$ is the cumulative amount of drug release, $M_{t}$ is the amount of drug released in time $t, M_{0}$ is the initial amount of drug in the material, and $k_{0}, k_{1}$, and $k_{H}$ are, respectively, the zeroorder, the first-order, and the Higuchi's release constant. The drug release mechanism was studied using the semiempirical Peppas model (Eq. (4)). ${ }^{22}$

$$
M_{t} / M_{0}=K t^{n}
$$

where $M_{t}$ is the amount of drug released in time $t, M_{0}$ is the initial amount of drug in the material, $K$ is the kinetic constant, and $n$ is the release exponent. The equation was applied to the first $60 \%$ of fractional release. For a cylindrical system, $n=0.45$ corresponds to Fickian diffusion (diffusion controlled), $n=0.89$ corresponds to non-Fickian (Case II transport), and values between 0.4 and 0.89 are referred to as anomalous transport.

\section{Results}

\section{RHEOLOGY}

The thermosensitive hydrogel, Lutrol F127 (copolymers of PEO-PPO-PEO), responds to both temperature and concentration changes. Therefore, the flow properties of Lutrol F127 as a function of temperature were assessed using rheology (Fig. 3a). At low temperatures, the formulation showed a typical fluid-like behavior, as indicated by the higher loss modulus than storage modulus $\left(G^{\prime}>G^{\prime}\right)$. As temperature increases, both the storage modulus $\left(G^{\prime}\right)$ and loss modulus $\left(G^{\prime \prime}\right)$ increases rapidly and the gelation temperature (where $G^{\prime}$ and $G^{\prime \prime}$ cross over) for $20 \%(\mathrm{w} / \mathrm{v}$ ) Lutrol F127 occurs around room temperature (RT $\left.\sim 25^{\circ} \mathrm{C}\right)$. The $G^{\prime}$, however, stays below $G^{\prime \prime}$ after the transition temperature, indicating that the material did not exhibit characteristics of a true gel. Figure $3 \mathrm{~b}$ compares the transition temperature of Lutrol F127 against concentration. For ease of interpretation, only the storage modulus $\left(G^{\prime}\right)$ is presented here. Clearly, the transition temperature shifts to lower temperature with increasing Lutrol F127 concentration with the $30 \% \mathrm{w} / \mathrm{v}$ formulation gelling at around $15^{\circ} \mathrm{C}$. On the other hand, $15 \% \mathrm{w} / \mathrm{v}$ Lutrol F127 simply behaved as a free flowing fluid, where $G^{\prime}$ remained low across all temperatures. As the purpose of an injectable drug delivery system in this case is to inject in the form of a liquid at room temperature that would gain strength in vivo, formulations of around $20 \%$ $\mathrm{w} / \mathrm{v}$ were more suitable for this scenario.

Cross-linking of alginate microspheres requires the use of $\mathrm{CaCl}_{2}$, therefore the behavior of Lutrol $\mathrm{F} 127$ with $\mathrm{CaCl}_{2}$ was examined by rheology and is shown in Fig. 4. In this case, a sudden increase in viscosity at a certain temperature would be indicative of the transition temperature of Lutrol F127 from solution to gel. The measurements showed that the presence of calcium ions in the solution shifts the transition temperature to lower temperatures along with an increase in viscosity. Formulation with the least amount of calcium ions was selected for further 

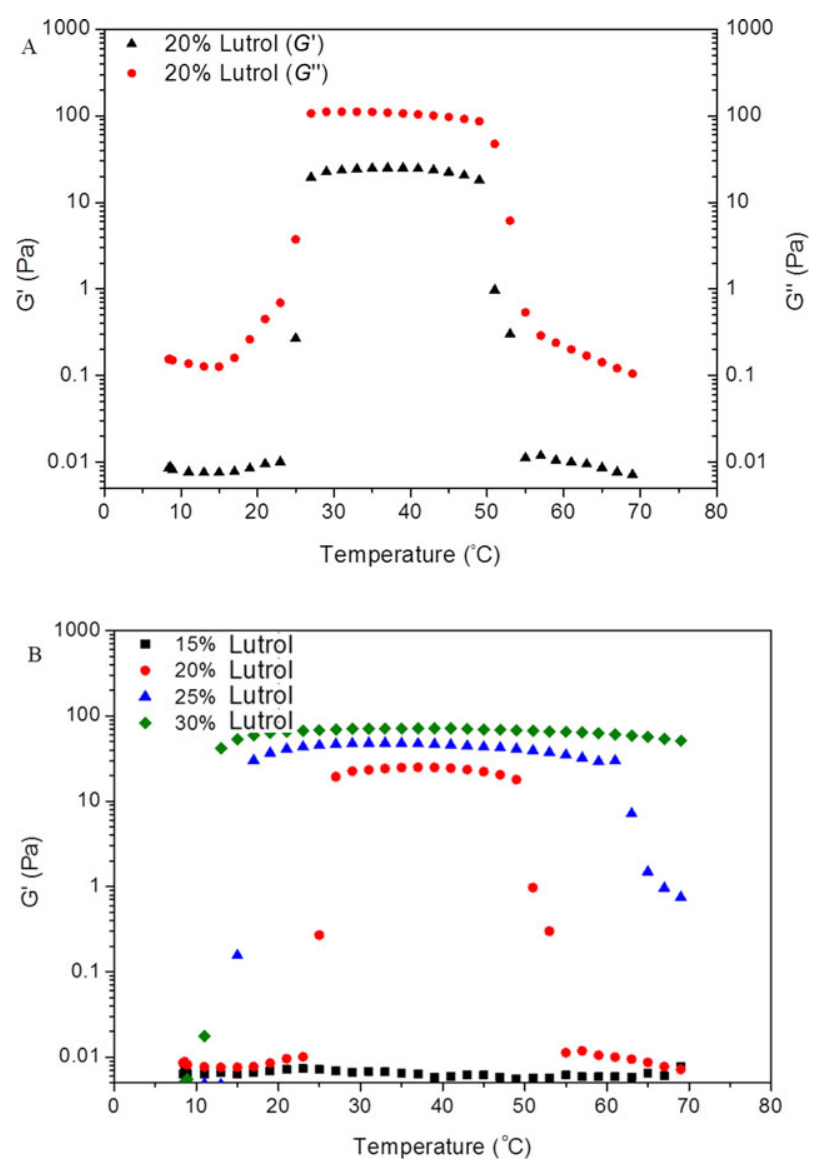

FIGURE 3. Rheological measurements. (A) Temperature sweep measurements of an aqueous solution of $20 \%$ (w/v) Lutrol F127. Transition temperature indicated by the temperature where $\mathrm{G}^{\prime}$ intersects $\mathrm{G}^{\prime \prime}$. (B) Temperature sweep measurements comparing various concentrations of Lutrol F127. Only the storage modulus ( $\left.G^{\prime}\right)$ was indicated for ease of interpretation.

investigation by varying the concentration of Lutrol F127, as shown in Fig. 5. The formulation with $20 \% \mathrm{w} / \mathrm{v}$ Lutrol F127 + $0.5 \% \mathrm{w} / \mathrm{v} \mathrm{CaCl}_{2}$ was found to have a viscosity higher than other concentrations at body temperature $\left(37^{\circ} \mathrm{C}\right)$, while still having a transition temperature of $\sim 25^{\circ} \mathrm{C}$. Therefore, this Lutrol F127 and $\mathrm{CaCl}_{2}$ combination were selected to be the most suitable concentration for injection.

\section{MICROSPHERE FABRICATION}

Alginate $(0.5 \% \mathrm{w} / \mathrm{v})$ solution was printed via inkjet into a cross-linking bath of 1,2 , or $5 \% \mathrm{w} / \mathrm{v} \mathrm{CaCl}_{2}$ to determine the optimum concentration for cross-linking as shown in Fig. 6. Images of microspheres cross-linked in $2 \% \mathrm{w} / \mathrm{v}$ and $5 \% \mathrm{w} / \mathrm{v} \mathrm{CaCl}_{2}$ bath were more circular and smaller as compared to spheres in $1 \%$ $\mathrm{w} / \mathrm{v} \mathrm{CaCl}_{2}$. Analysis of the sphere diameter was converted into a frequency distribution histogram and showed that increasing cross-linking concentration leads to smaller average sphere diameters and consistency (diameter decreases from 37.9 to 12.2 $\mu \mathrm{m}$ for $1 \% \mathrm{w} / \mathrm{v}$ and $5 \% \mathrm{w} / \mathrm{v} \mathrm{CaCl}_{2}$, respectively). Lower dispersion across the histogram indicated that the microspheres in higher concentration baths were more consistent in size. Based on these observations, the $2 \% \mathrm{w} / \mathrm{v} \mathrm{CaCl}_{2}$ was identified to be the minimum cross-linking concentration required for this inkjet printing setup to obtain microspheres with narrow size distribution.

\section{IN VITRO DRUG RELEASE STUDIES}

The cumulative release and mass loss profile of $20 \% \mathrm{w} / \mathrm{v}$ Lutrol F127 are shown in Fig. 7. In $24 \mathrm{~h}$, the release of the model drug (FITC-dextran) reached completion and the percentage material remaining was $10 \%$. This showed that the material is susceptible to hydrolysis and the drug release is not only via mechanisms of diffusion, but also through erosion. The release kinetics of samples was evaluated using the zero-order, first-order, and Higuchi's equations as shown in Table I. The coefficient of determination $\left(r^{2}\right)$ for each sample is displayed in the table with the $r^{2}$ closest to unity underlined. The highest $r^{2}$ values for all samples were in the Higuchi's model, indicating that the release of the model from all system was a diffusion-dependent process. Based on the power law equation, the values of $n$ were

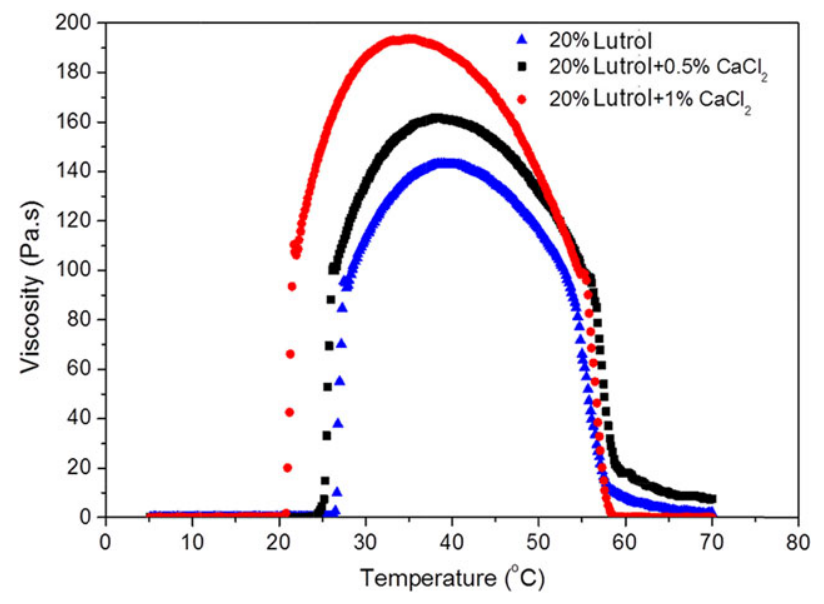

FIGURE 4. Temperature ramp measurements of $20 \%$ (w/v) Lutrol $\mathrm{F} 127$ with various amounts of $\mathrm{CaCl}_{2}$ added.

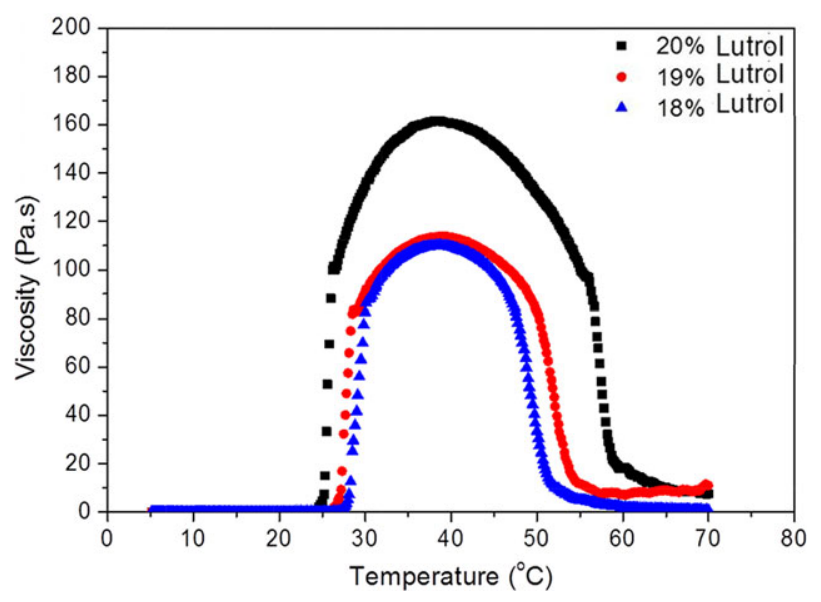

FIGURE 5. Temperature ramp measurements with various concentration of Lutrol F127 added to $0.5 \%(\mathrm{w} / \mathrm{v}) \mathrm{CaCl}_{2}$. 

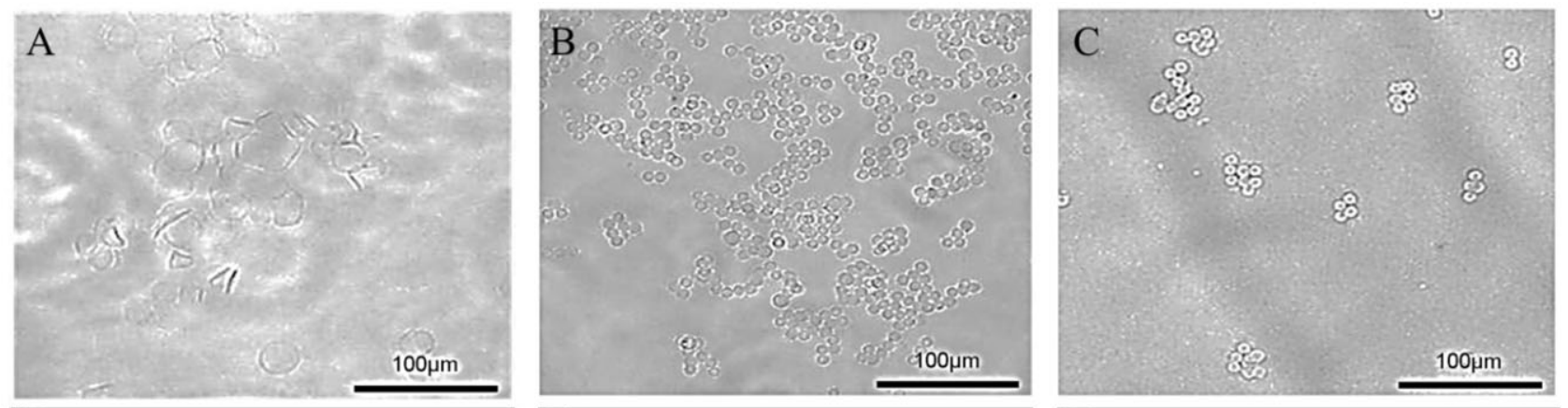

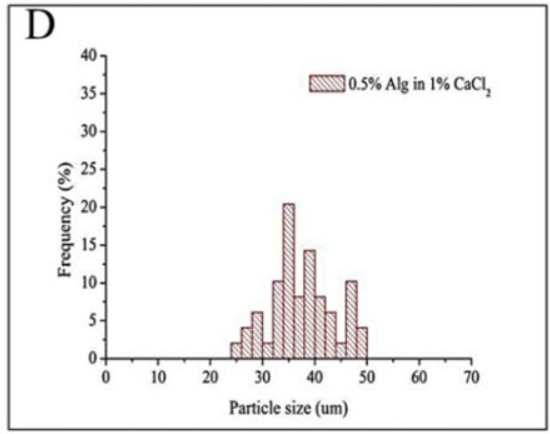

Average microsphere diameter- 37.9um

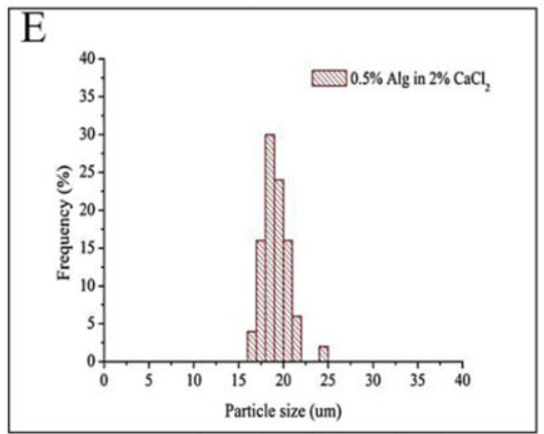

Average microsphere diameter- 19.5 um

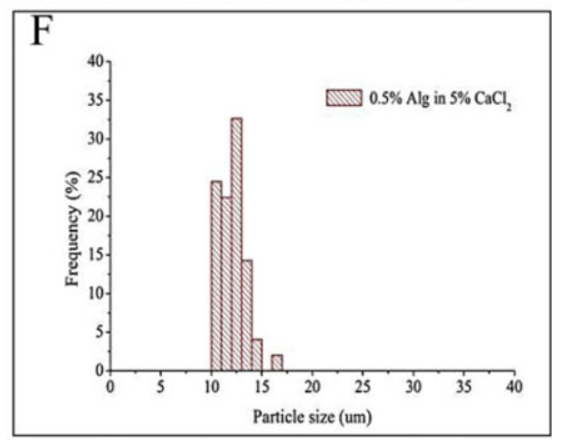

Average microsphere diameter- $12.2 \mathrm{um}$

FIGURE 6. Images of alginate microspheres printed in cross-linking baths of $1,2,5 \%(\mathrm{w} / \mathrm{v}) \mathrm{CaCl}_{2}(\mathrm{~A}-\mathrm{C})$. Frequency distribution histograms of $0.5 \%$ $(\mathrm{w} / \mathrm{v})$ in various $\mathrm{CaCl}_{2}$ baths (D-F).

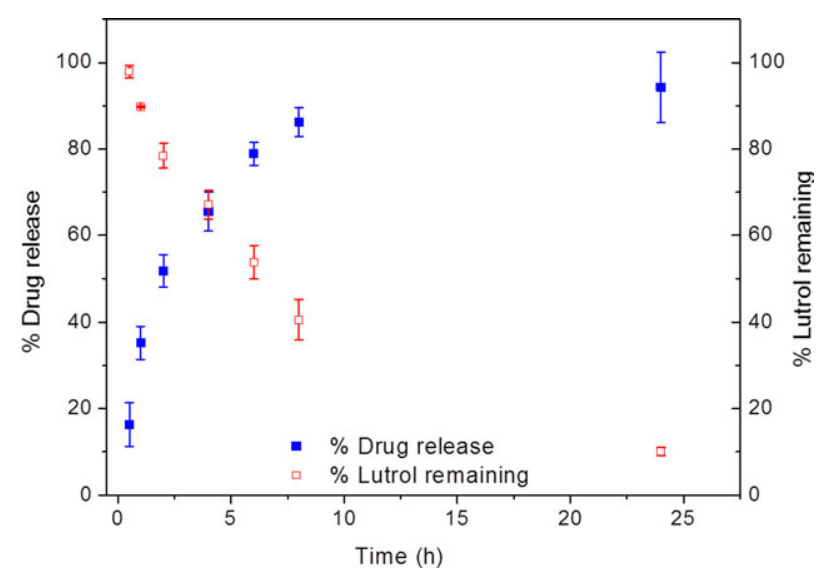

FIGURE 7. Cumulative release profile of $20 \%$ (w/v) Lutrol F127 incorporated with model drug over $24 \mathrm{~h}(n=3)$. Data represents mean \pm SD.

TABLE I Coefficient of Determination

\begin{tabular}{lcccc}
\hline System & $\begin{array}{c}\text { Zero Order } \\
r^{2}\end{array}$ & $\begin{array}{c}\text { First Order } \\
r^{2}\end{array}$ & $\begin{array}{c}\text { Higuchi } \\
r^{2}\end{array}$ & $\begin{array}{c}\text { Peppas } \\
n\end{array}$ \\
\hline Lutrol F127 & 0.570 & 0.407 & $\underline{0.968}$ & 0.693 \\
$\begin{array}{l}\text { Microspheres only } \\
\begin{array}{l}\text { 100,000 } \\
\text { microspheres/ }\end{array}\end{array}$ & 0.944 & 0.470 & $\underline{\underline{0.989}}$ & - \\
$\quad$ mL Lutrol F127 & 0.905 & 0.477 & $\underline{0.987}$ & 0.626 \\
$\begin{array}{l}500,000 \\
\quad \text { microspheres/ }\end{array}$ & 0.887 & 0.505 & $\underline{0.977}$ & 0.608 \\
$\quad$ mL Lutrol F127 & & & & \\
\hline
\end{tabular}

all between 0.45 and 0.89 ( $n=0.693$ for pristine Lutrol F127, $n=0.626$ and 0.608 for Lutrol F127 loaded with 100,000 and 500,000 microspheres $/ \mathrm{mL}$, respectively), indicating that the release mechanism was a combination of Fickian diffusion and Case II transport. This suggested that the drug not only diffuses through the polymer matrix but also through the material eroding. The model was not applied for the release profile of alginate microspheres as the burst release was greater than $60 \%$ within the first $30 \mathrm{~min}$.

For drug release of alginate microspheres, the release reached completion in $6 \mathrm{~h}$ along with a very large burst effect, as shown in Fig. 8. This suggested that the drug release has already taken place the moment the spheres came in contact with the crosslinking solution after ejection. In comparison, by printing microspheres into a cross-linking solution containing the drug followed by the addition of Lutrol F127, the release profile became more sustained. Incorporating 100,000 microspheres/mL into Lutrol F127 showed a lower burst effect in the first $30 \mathrm{~min}$ and released only $45 \%$ as compared to $94 \%$ for spheres without being embedded inside Lutrol F127. The release profile of Lutrol F127 with microspheres was also more sustained than pristine Lutrol F127 (Fig. 7) and released only 70\% in $24 \mathrm{~h}$. By increasing the number of microspheres within Lutrol F127 to 500,000 microspheres $/ \mathrm{mL}$ while keeping the total mass of drug per sample unchanged $(2 \mu \mathrm{g} / \mathrm{mL})$, the amount of drug released at every time point was further reduced. The release from Lutrol F127 with 100,000 microspheres $/ \mathrm{mL}$ was around $70 \%$ and $80 \%$ after 1 and 5 days, respectively, whereas Lutrol F127 with 500,000 microspheres $/ \mathrm{mL}$ released around $63 \%$ and $75 \%$ after 1 and 5 days, respectively. While no significant difference was observed between release profiles of Lutrol F127 with 100,000 microspheres/mL and Lutrol F127 with 500,000 microspheres/mL, in both cases 


\section{RESEARCH ARTICLE}

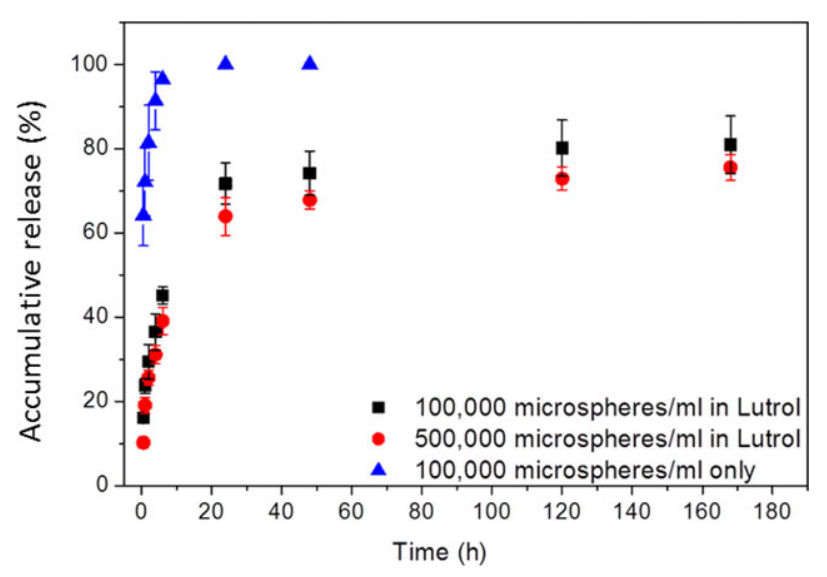

FIGURE 8. Cumulative release profiles of alginate microspheres incorporated with model drugs in saline $(100,000$ microspheres only) and dispersed in $20 \%(\mathrm{w} / \mathrm{v})$ Lutrol F127 $(n=3)$. Data represents mean $\pm \mathrm{SD}$.

the release was considerably prolonged compared to beads only and Lutrol F127 only cases.

\section{Discussion}

Injectable hydrogels have become an attractive approach to delivery drugs locally as compared to traditional implantable drug delivery systems. The combination of microspheres and injectable hydrogels can result in a prolonged drug release profile not seen when these systems are used individually. With the addition of using an inkjet printing system to fabricate microspheres, the steps to prepare an injectable system with microsphere carriers can be largely reduced. This is in comparison to common procedures, where the fabrication of drug carrying carriers and the matrix polymer were prepared separately in multiple steps. ${ }^{13,16,23}$

Lutrol F127 was selected as the drug carrier for injectable applications due to its reversible sol-gel transition behavior and ability to incorporate both hydrophilic and hydrophobic substances that are insoluble in aqueous solution. It is well documented in the literature that both micellization and gelation depend on different factors, namely, temperature and polymer concentration. ${ }^{24}$ This is consistent with the observation from rheological measurements that an increase in polymer concentration leads to an increase in viscosity but also a lowering of gelation temperature.

Prior to reaching the critical micellar concentration (CMC), Lutrol F127 exists as individually monomolecular micelles at low concentrations. As the concentration increases, a phase transition from a free-flowing solution to a gel-like structure occurs at a certain temperature, referred to as the critical micellar temperature (CMT). This was clearly observed when comparing between 15\% w/v Lutrol F127 and samples greater than 20\% w/v. The 15\% w/v Lutrol F127 did not show a substantial increase in storage modulus across the range of temperatures tested as it did not reach the $\mathrm{CMC}$ necessary to induce aggregation at higher temperatures. Lutrol F127, however, did not exhibit a "true gel" behavior as indicated by the lower $G^{\prime}$ than $G^{\prime \prime}$ beyond the transition temperature. It has been reported that the formation of gel-like characteristics was due to the progressive dehydration of the PPO core and formation of multimolecular clusters with the hydrophilic PEO facing outwards. ${ }^{10,11}$ Therefore, it is possible that the aggregation of micelles could increase the viscosity, allowing it to behave like a gel but is still not indicative of a "true gel." In addition, it is necessary to note that the increase in polymer concentration also results in a reduction of CMT, which can lead to difficulty in handling at room temperature. Therefore, the $20 \% \mathrm{w} / \mathrm{v}$ Lutrol F127 was selected as the concentration for further characterization.

To fabricate alginate microspheres, a cross-linking solution is usually required. Therefore, it was necessary to examine the effect of ions on the gelation temperature of Lutrol F127, as the microspheres in the cross-linking solution will be combined with Lutrol F127. The addition of divalent cations, in this case $\mathrm{CaCl}_{2}$, shifted the transition temperature of Lutrol F127 to lower temperatures. The lowering of transition temperature was proportional to the increase in calcium concentration. Studies into the effects of various salts on the transition temperature of F127 have been conducted by Pandit et al. ${ }^{25,26}$ The lowering of transition temperature can be explained by the dehydration of the PPO block, lowering the solubility and favoring micellization. ${ }^{25}$ As a result, both the CMC and CMT will be lowered since a gel containing these salts at any given temperature will consist of micelles that are more closely packed than gels without salt. ${ }^{26}$

Fabrication of alginate microspheres using a nozzle-based ink-jetting system enables the production of uniform drop sizes with good control, which otherwise can affect the reliability of drug release results from inconsistent particle size. Sodium alginate was selected as the microsphere matrix material in this study for its ease of gelation, mechanical stability, and good tissue compatibility. ${ }^{19}$ The printing of alginate microspheres into various concentrations of $\mathrm{CaCl}_{2}$ indicated that the use of higher concentration baths leads to smaller and more circular beads. This is consistent with the literature where the generation of particles improves with $\mathrm{CaCl}_{2}$ concentration. ${ }^{18}$ In this study, a lower alginate concentration $(0.5 \% \mathrm{w} / \mathrm{v})$ was used as compared to studies also printing alginate. This was due to the higher chances of clogging at the nozzle when higher concentrations $(>0.5 \% \mathrm{w} / \mathrm{v})$ were used. In addition, studies printing with concentrations greater than $1 \% \mathrm{w} / \mathrm{v}$ use either a nozzle size much larger than the cartridge used in this study $(21 \mu \mathrm{m}),{ }^{18}$ or by adapting a commercial inkjet system that can eject high viscous solution up to $100 \mathrm{mPa} / \mathrm{s}^{27}$ Combining this result with the previous findings on Lutrol $\mathrm{F} 127$ and $\mathrm{CaCl}_{2}$ concentrations, the $2 \%$ $\mathrm{w} / \mathrm{v} \mathrm{CaCl} \mathrm{Cl}_{2}$ bath was found to be the minimal concentration for printing and was diluted with Lutrol F127 to yield a final concentration of $20 \% \mathrm{w} / \mathrm{v}$ Lutrol $\mathrm{F} 127+0.5 \% \mathrm{w} / \mathrm{v} \mathrm{CaCl}_{2}$ for drug delivery.

The in vitro drug release of FITC-dextran from $20 \% \mathrm{w} / \mathrm{v}$ Lutrol F127 reached completion in $24 \mathrm{~h}$, along with a continuous decrease in polymer mass at each measured time point. This indicated that release of drugs from Lutrol F127 was not only through the mechanism of diffusion but also through erosion of the polymer matrix. ${ }^{11,28}$ A method to sustain the release was through the addition of microspheres within the drug containing polymer matrix. It was observed that the incorporation of 
microspheres into Lutrol F127 has decreased not only the burst release, but further sustained the total release compared to pristine Lutrol F127 and drug-loaded microspheres alone. This is consistent with studies in the literature where the incorporation of drug-loaded microparticles/nanoparticles in polymer matrices has decreased the burst effect compared to their individual counterparts. ${ }^{23}$ The drug loading capacity for this system was selected after considering the solubility and detection limit of FITC-dextran and can be simply increased or decreased depending on the required therapeutic window and drug solubility.

By combining the two components together, Lutrol F127 can act as an additional diffusion pathway for the drugs in the microspheres and drug release can be further sustained. In addition, since both Lutrol F127 and alginate microspheres now contain drugs, the concentration gradient between these two will become smaller, allowing the release of drugs from microspheres to be also dependent on the dissolution of Lutrol F127. A similar concept was reported by Giovagnoli et al., ${ }^{16}$ where the release of doxycycline from alginate microparticles embedded in F127 was $80 \%$ in 3 days. Their system however was added with a thickening agent to further increase the stability of Lutrol F127 and only benefited the release profile during earlier time points. By adapting a nozzle-based ink jetting system to fabricate microspheres, this injectable formulation can be subjected to simple adjustments, such as sphere quantity, size, cross-linking solution, and concentration of hydrogel. These have the potential to alter the release profile as seen by the release profile when microsphere quantity was increased from 100,000 to $500,000 \mathrm{mi}$ crospheres $/ \mathrm{mL}$.

It is also noteworthy that the current drug release setup may not accurately represent the conditions in the middle ear. Studies conducted by Wang et al., ${ }^{5,6}$ have reported longer release times using pristine $17 \%$ Lutrol F127, where the polymer delivered dexamethasone up to 10 days when injected intratympanically. The in vitro release studies were, however, conducted on snapwells consisting of a $0.4 \mu \mathrm{m}$ pore polycarbonate membrane. Within the middle ear, Lutrol F127 will not be in direct contact with the perilymph (with diffusion through round window membrane, and also the stapes as has recently been demonstrated by O'leary and co-workers). ${ }^{29,30}$ Therefore, the concentration gradient will be lower and the dissolution of the polymer will be lower too. In addition, as the perilymph effectively does not flow, which is a very different situation to the rotating fluid on an orbital shaker, it is believed that the current system when injected in vivo could be more sustained. ${ }^{31}$ Further studies will be conducted using a Franz-type diffusion cells that is often used to study drug release through skin. ${ }^{32-34}$ Collectively, this work has identified that $20 \% \mathrm{w} / \mathrm{v}$ Lutrol F127 with $0.5 \% \mathrm{w} / \mathrm{v} \mathrm{CaCl}_{2}$ formulations with alginate microspheres provides an excellent substrate for injectable drug delivery purposes, while maintaining processability and can be easily modified to alter the drug release profile.

\section{Conclusions}

An injectable drug delivery system for the middle ear was explored by embedding alginate microspheres into a thermosensi- tive gel, Lutrol F127. In addition, a nozzle-based printing system was assessed as an alternative method to fabricate hydrogel microspheres that can largely simply be the formulation process of the injectable delivery system. The optimum combinations of polymer and cross-linking conditions of each component were determined by rheology and microscopy. In vitro drug release of a model drug (FITC-dextran) from pristine Lutrol F127 and alginate microspheres both reached completion in less than 24 h. Interestingly, the incorporation of microspheres within Lutrol F127 showed sustained release and a lower burst effect due to the presence of a secondary barrier to diffusion and a lower concentration gradient. The profile is likely to be further sustained in vivo due to the less extreme sink conditions and future studies will first look into experimenting with a Franz-type diffusion cell setup. The approach presented in this study demonstrated a simple route to formulate injectable hydrogel-containing microspheres, where the release profile can be altered easily through changes in printing parameters and condition.

\section{Acknowledgments}

This research was supported by the Australian Research Council, Super science Fellowship Scheme, ARC Centre for Electromaterials Science (ACES). The authors would like to acknowledge the Australian National Fabrication Facility (ANFF) for funding of the equipments, Dr. David Sly for initial testing of material in Guinea pigs, Dr. Bo Weng for initial training on Dimatix inkjet printer, and ARC Fellowships to Gordon G. Wallace (Australian Laureate Fellow). Sina Naficy acknowledges UOW Global Challenges program for their support.

\section{References}

1. Isaacson, J. E.; Vora, N. M. Am Fam Physician 2003, 68(6), 1125-1132.

2. Raphael, Y. Br Med Bull 2002, 63(1), 25-38.

3. Nadot, J. B. N Engl J Med 1993, 329, 1092-1102.

4. Staecker, H.; Rodgers, B. Expert Opin Drug Delivery 2013, 10, 639-650.

5. Wang, X.; Dellamary, L.; Fernandez, R.; Harrop, A.; Keithley, E. M.; Harris, J. P.; Ye, Q.; Lichter, J.; LeBel, C.; Piu, F. Audiol Neurotol 2009, 14(6), 393-401.

6. Wang, X.; Dellamary, L.; Fernandez, R.; Ye, Q.; Lebel, C.; Piu, F. Laryngoscope 2011, 121, 385-391.

7. Borden, R. C.; Saunders, J. E.; Berryhill, W. E.; Krempl, G. A.; Thompson, D. M.; Queimado, L. Audiol Neurotol 2011, 16, 1-11.

8. Nguyen, M. K.; Lee, D. S. Macromol Biosci 2010, 10(6), 563-579.

9. Yu, L.; Ding, J. Chem Soc Rev 2008, 37(8), 1473-1481.

10. Escobar-Chávez, J. J.; López-Cervantes, M.; Naïk, A.; Kalia, Y. N.; QuintanarGuerrero, D.; Ganem-Quintanar, A. J Pharm Pharm Sci 2006, 9(3), 339-358.

11. Nie, S.; Hsiao, W. L.; Pan, W.; Yang, Z. Int J Nanomed 2011, 6, 151-166.

12. Lippens, E.; Swennen, I.; Gironès, J.; Declercq, H.; Vertenten, G.; Vlaminck, L.; Gasthuys, F.; Schacht, E.; Cornelissen, R. J Biomater Appl 2013, 27(7), 828-839.

13. Jannin, V.; Pochard, E.; Chambin, O. Int J Pharm 2006, 309(1-2), 6-15.

14. Gong, C.; Shi, S.; Dong, P.; Zheng, L.; Fu, S.; Guo, G.; Yang, J. L.; Wei, Y. Q.; Qian, Z. Y. BMC Biotechnol 2009, 9(1), 1-13.

15. Kretlow, J. D.; Klouda, L.; Mikos, A. G. Adv Drug Delivery Rev 2007, 59(4-5), 263-273. 


\section{RESEARCH ARTICLE}

16. Giovagnoli, S.; Tsai, T.; DeLuca, P. AAPS PharmSciTech 2010, 11(1), 212 220.

17. Desai, S.; Perkins, J.; Harrison, B. S.; Sankar, J. Mater Sci Eng, B 2010, 168(1-3), 127-131.

18. Herran, C. L.; Huang, Y. J Manuf Process 2012, 14(2), 98-106.

19. Cohen, D. L.; Lo, W.; Tsavaris, A.; Peng, D.; Lipson, H.; Bonassar, L. J. Tissue Eng, Part C 2011, 17(2), 239-248.

20. Schmolka, I. R. Tissue Eng, Part C 1972, 6(6), 571-582.

21. Costa, P.; Lobo, J. M. S. J Pharm Sci 2001, 13, 123-133.

22. Ritger, P. L.; Peppas, N. A. J Controlled Release 1987, 5, 23-36.

23. Gou, M.; Li, X.; Dai, M.; Gong, C.; Wang, X.; Xie, Y.; Deng, H. X.; Chen, L. J.; Zhao, X.; Qian, Z. Y.; Wei, Y. Q. Int J Pharm 2008, 359(1-2), 228-233.

24. Bonacucina, G.; Cespi, M.; Mencarelli, G.; Giorgioni, G.; Palmieri, G. F. Polymers 2011, 3(2), 779-811.

25. Pandit, N.; Trygstad, T.; Croy, S.; Bohorquez, M.; Koch, C. J Colloid Interface Sci 2000, 222(2), 213-220.
26. Pandit, N. K.; Kisaka, J. Int J Pharm 1996, 145(1-2), 129-136.

27. Iwanaga, S.; Saito, N.; Sanae, H.; Nakamura, M. Colloids Surf, B 2013, 109, 301-306.

28. Xuan, J.-J.; Yan, Y.-D.; Oh, D. H.; Choi, Y. K.; Yong, C. S.; Choi, H.-G. Drug Deliv 2011, 18(5), 305-311.

29. Salt, A. N.; King, E. B.; Hartsock, J. J.; Gill, R. M.; O’Leary, S. J. Hearing Res 2012, 283(1-2), 14-23.

30. King, E. B.; Salt, A. N.; Kel, G. E.; Eastwood, H. T.; O'Leary, S. J. Hearing Res 2013, 304, 159-166.

31. Salt, A. N. Otol Neurotol 2008, 29(3), 401-406.

32. Shim, J.; Seok Kang, H.; Park, W.-S.; Han, S.-H.; Kim, J.; Chang, I.-S. J Controlled Release 2004, 97(3), 477-484.

33. Thakur, R. A.; Florek, C. A.; Kohn, J.; Michniak, B. B. Int J Pharm 2008, 364(1), 87-93.

34. Parsaee, S.; Sarbolouki, M. N.; Parnianpour, M. Int J Pharm 2002, 241(1), 185 190. 